\title{
Digestion des protéines de pois et de soja chez le veau préruminant. II. Digestibilité apparente à la fin de l'iléon et du tube digestif
}

\author{
I. Nunes Do Prado 1, R. Toullec 1, P. Guilloteau ${ }^{1}$ et J. Guéguen 2 \\ avec la collaboration technique de M. Beaufils, S. Boussion, M. Connan, M. Formal, \\ $J$. Lareynie et $Y$. Manis
}

1 INRA, Laboratoire du jeune ruminant, 65, rue de Saint-Brieuc, 35042 Rennes cédex;

2 INRA, Laboratoire de biochimie et technologie des protéines, rue de la Géraudière, 44072 Nantes cédex 03, France

(reçu le 14 novembre 1988, accepté le 6 juin1989)

Résumé - Trois laits de remplacement (témoin, pois et soja) sont distribués à 6 veaux préruminants munis d'une canule réentrante à la fin de l'iléon. Dans l'aliment témoin, les protéines sont fournies presque exclusivement par de la poudre de lait écrémé. Dans l'aliment pois, 33,5\% d'entre elles sont apportées par une farine de pois dépelliculé prégélatinisée et le reste par de la poudre de lait écrémé. Dans l'aliment soja, elles proviennent pour $73,2 \%$ d'un isolat de soja et pour le reste de la poudre de lactosérum. La digestibilité apparente de l'azote total est moins élevée avec les aliments pois et soja qu'avec l'aliment témoin $(0,92,0,91$ et 0,95 à la fin de l'iléon; 0,92 , 0,94 et 0,97 à la fin du tube digestif). La digestibilité iléale de la plupart des acides aminés est également abaissée, surtout celle de la cystine avec l'aliment pois $(-0,06$; non significatif) et celle de la thréonine avec l'aliment soja $(-0,07 ; P<0,01)$. Quel que soit le régime, les protéines des digesta iléaux semblent être principalement d'origine endogène et bactérienne. Bien que de faibles quantités de protéines alimentaires partiellement dégradées puissent échapper à la digestion dans l'intestin grêle dans le cas des aliments pois et soja, la digestibilité réelle des protéines étudiées est probablement très élevée.

protéines — digestion — iléon - veau préruminant

Summary - Digestion of pea and soya-bean proteins in preruminant calves. II. lleal and faecal apparent digestibilities. Three milk-substitutes (control, pea and soya-bean) were given to 6 preruminant calves fitted with re-entrant canulas in the terminal ileum (Table I). In the control diet, protein was almost entirely provided by skim-milk powder. In the pea diet, a pregelatinized dehulled pea flour provided $33.5 \%$ of the protein, the remainder being supplied by skim-milk powder. In the soya-bean diet $73.2 \%$ of the protein were provided by a soya-bean isolate and the remainder by whey powder. The apparent digestibility of total nitrogen was significantly lower with the pea and soya-bean diets than with the control diet $(0.92,0.91$ and 0.95 at the end of the ileum, 0.92, 0.94 
and 0.97 at the end of the whole digestive tract, respectively). Also the ileal digestibility of most amino acids decreased with the pea and soya-bean diets; the differences were greatest for cystine $(-0,06$; non significant) with the pea diet and for threonine $(-0.07 ; P<0.01)$ with the soya-bean diet. Irrespective of the diet the protein escaping digestion in the small intestine appeared to be mainly from endogenous and bacterial origin. Although small amounts of partially degraded dietary protein could be present in ileal digesta with the pea and soya-bean diets, their true digestibility was probably very high.

proteins - digestion - ileum - preruminant calf

\section{INTRODUCTION}

Les jeunes mammifères présentent parfois des manifestations d'intolérance aux protéines de substitution introduites dans le régime lacté. Ces problèmes sont particulièrement importants chez le bébé (Walker-Smith, 1984) et chez le veau préruminant (Toullec et al., 1983). La réduction récente de la production de poudre de lait écrémé a rendu nécessaire la diversification des sources de protéines incorporables dans les aliments d'allaitement destinés à ce dernier. Les légumineuses sont les sources de protéines végétales les plus abondantes. Plusieurs produits issus du soja sont préparés industriellement, tandis que le pois commence à être l'objet de traitements technologiques susceptibles d'améliorer son utilisation. Si de nombreuses données ont été publiées sur la digestion des tourteaux et des concentrats de soja (Paruelle et al., 1972; Guilloteau et al., 1986), il n'en est pas de même pour les isolats de soja et les pois traités. L'analyse des fèces fournit moins d'information que celle des digesta iléaux sur la digestibilité des acides aminés et l'origine des protéines indigérées, à cause des remaniements importants entraînés par le développement de la flore dans le gros intestin (Guilloteau et al., 1980 et 1986). Ainsi, chez le veau nourri au lait, l'examen de la composition en acides aminés des protéines des digesta iléaux indique qu'elles sont principalement d'origine endogène. En revanche, le peptidoglycane de la paroi de Pseudomonas methylotropha échappe en totalité à la digestion dans l'intestin grêle. De même, la glycinine du soja et les solubles de poisson semblent être partiellement indigérés chez des veaux recevant des aliments contenant un concentrat protéique de soja ou un hydrolysat de poisson.

Le but de ce travail a été d'élargir ces observations en étudiant l'influence du remplacement des protéines du lait par celles d'une farine de pois décortiqué prégélatinisée ou d'un isolat de soja sur les digestibilités iléale et fécale. D'autres résultats concernant les taux circulants de nutriments, la formation d'anticorps contre les protéines alimentaires et la perméabilité intestinale aux macromolécules sont rapportés dans une autre publication (Nunes do Prado et al., 1989).

\section{MATÉRIEL ET MÉTHODES}

\section{Aliments, animaux, régimes et prélè- vements}

La composition globale des sources de protéines étudiées et des aliments, ainsi que 
les caractéristiques des veaux et de leur conduite, ont été rapportées en détail dans une publication antérieure (Nunes do Prado et al., 1989). Brièvement, 3 aliments (témoin, pois et soja), contenant environ $25 \%$ de matières azotées et $20 \%$ de matières grasses par rapport à la matière sèche, ont été comparés. Dans l'aliment témoin, les protéines étaient fournies en quasi-totalité par de la poudre de lait écrémé. Dans l'aliment pois, elles provenaient pour 33,5\% d'une farine de pois dépelliculé prégélatinisée, le reste étant apporté par de la poudre de lait écrémé. Dans l'aliment soja, elles étaient fournies en majeure partie $(73,5 \%)$ par un isolat de soja, le reste provenant de la poudre de lactosérum. Ces aliments avaient des teneurs quasiment identiques en calcium, magnésium, sodium et potassium; cependant, les aliments pois et soja conte- naient davantage de phosphore $(0,84$ et $0,89 \%$ de la matière sèche, au lieu de 0,72 ). La composition en acides aminés des sources de protéines étudiées et des aliments est rapportée dans le Tableau I.

Six veaux mâles munis d'une canule réentrante à la fin de l'iléon ont reçu chacun des 3 aliments dans un ordre différent, au cours de 3 périodes successives de 3 semaines (1 d'adaptation, 1 de mesure de la digestibilité fécale et 1 de mesure de la digestibilité iléale). Les fèces ont été collectées en totalité tous les matins pendant 5 j consécutifs. Après pesée, une partie aliquote a été prélevée et transférée à $-18{ }^{\circ} \mathrm{C}$, dans un récipient par veau et par période. Les canules réentrantes ont été déconnectées $2 \mathrm{j}$ avant le début de la récolte des digesta iléaux. Ceux-ci ont été recueillis en permanence dans des récipients

Tableau I. Composition en acides aminés (AA) des sources de protéines et des aliments (\% des acides aminés dosés).

$A A$

Source protéique

Pois Soja

A. aspartique

Thréonine

Sérine

A. glutamique

Proline

Glycine

Alanine

Valine

Cystine

Méthionine

Isoleucine

Leucine

Tyrosine

Phénylalanine

Lysine

Histidine

Arginine

$\begin{array}{rr}12,35 & 11,16 \\ 3,74 & 3,65 \\ 4,38 & 4,56 \\ 18,43 & 20,15 \\ 4,07 & 5,28 \\ 4,76 & 4,28 \\ 4,92 & 4,43 \\ 5,58 & 5,74 \\ 1,50 & 1,34 \\ 1,20 & 1,37 \\ 4,81 & 5,32 \\ 7,70 & 8,19 \\ 3,09 & 3,61 \\ 4,83 & 5,17 \\ 7,37 & 6,13 \\ 2,45 & 2,49 \\ 8,81 & 7,12\end{array}$

$100,45 \quad 108,98$

Somme des AA dosés

$(\mathrm{g} / 16 \mathrm{~g} \mathrm{~N})$

$N$ des AA dosés
$88,39 \quad 92,17$

(\% de $\mathrm{N}$ total) 1

$1 \mathrm{~N}$ amidé de l'asparagine et de la glutamine exclu.

\section{Aliment}

Témoin Pois Soja

$\begin{array}{rrr}7,61 & 9,42 & 10,94 \\ 4,73 & 4,41 & 4,45 \\ 4,64 & 4,68 & 4,57 \\ 20,94 & 19,31 & 19,34 \\ 9,66 & 7,48 & 5,38 \\ 1,82 & 2,81 & 3,74 \\ 3,06 & 3,68 & 4,45 \\ 6,95 & 6,13 & 5,76 \\ 0,94 & 1,08 & 1,55 \\ 3,10 & 3,03 & 2,83 \\ 5,59 & 5,09 & 5,42 \\ 9,10 & 9,05 & 8,41 \\ 4,16 & 4,03 & 3,16 \\ 4,41 & 4,54 & 4,61 \\ 7,51 & 7,72 & 7,16 \\ 2,48 & 2,53 & 2,29 \\ 3,31 & 5,01 & 5,93\end{array}$

$106,70 \quad 106,39 \quad 105,58$

$84,21 \quad 87,11 \quad 87,91$ 
contenant un cristal de thymol, pendant $4 j$ consécutifs. Les récipients de collecte ont été changés après chaque repas. Pour chaque veau, les digesta ramassés le soir ont été mis à $+4^{\circ} \mathrm{C}$ jusqu'au leridemain matin, où ils ont été mélangés avec ceux obtenus pendant la nuit. L'ensemble a été pesé et homogénéisé, puis une partie aliquote a été transférée à - $18^{\circ} \mathrm{C}$ dans un récipient par veau et par période.

\section{Analyses et calculs}

Les digesta et les fèces ont été lyophilisés. Dans les échantillons de farine de pois, d'isolat de soja, d'aliments, de digesta et de fèces, les teneurs en matière sèche, cendres, azote et acides aminés ont été mesurées selon les méthodes utilisées par Guilloteau et al. (1986). La teneur en lipides a été déterminée par extraction à l'éther éthylique, après hydrolyse, à l'aide d'un appareil Soxhlet. Le calcium, le magnésium, le potassium et le sodium ont été dosés par spectrophotométrie d'absorption atomique et le phosphore par la méthode colorimétrique au vanadomolybdate. Les analyses d'acides aminés ont été effectuées dans des échantillons moyens de farine de pois, d'isolat de soja, d'aliment témoin et de chaque complément ayant servi à préparer les aliments pois et soja. Pour les fèces, un échantillon moyen par régime a été constitué en mélangeant les échantillons obtenus pour chaque veau, au prorata de leur teneur en azote. En revanche, l'analyse des acides aminés a été pratiquée sur tous les échantillons individuels de digesta. La légumine immunoréactive a été recherchée dans le pois, les fèces et les digesta par la technique ELISA en "sandwich indirect" décrite par Nunes do Prado et al. (1989). Pour cela, les protéines ont été extraites par du tampon phosphate de $\mathrm{pH}$ 7,4 (Guéguen et al., 1984).

Pour l'ensemble des critères étudiés, les effets du régime ont été soumis à une analyse de variance et les moyennes classées à l'aide du test de Newman \& Keuls. Les compositions en acides aminés des digesta et des fèces ont été comparées entre elles et à celles de protéines endogènes, alimentaires et bactériennes, en calculant la distance du $\chi^{2}$ et par analyse factorielle des correspondances (Guilloteau et al., 1983). Comme exemples de compositions de protéines endogènes, nous avons choisi celles des fèces d'agneau axénique (Combe, 1976) et de méconium de veau (Grongnet et al., 1981). La moyenne des compositions en acides aminés des bactéries fécales de porc (Mason et al., 1976) et de mouton (Mason, 1979) a servi de modèle pour la composition des protéines bactériennes du tube digestif. Pour les protéines alimentaires, en plus de nos propres résultats d'analyse, nous avons utilisé les données publiées sur la légumine, la viciline (Guéguen et al., 1984), la conviciline (Croy et al., 1980) et les albumines du pois (Gwiazda et al., 1980), ainsi que la glycinine et la $\beta$-conglycinine du soja (Wolf, 1972).

\section{RÉsultats}

\section{Appétit, croissance et état sanitaire}

Les aliments ont été généralement bien consommés, sauf l'aliment soja chez l'un des veaux qui n'a ingéré que $79 \%$ de la quantité offerte, après la fin de la transition alimentaire. En moyenne, les refus ont été de $1 \pm 1,3 \pm 2$ et $6 \pm 3 \%$ avec les aliments témoin, pois et soja, respectivement. L'état sanitaire des veaux a été satisfaisant tout au long de l'essai. Les teneurs en matière sèche des fèces ont été très voisines avec les trois régimes pendant les périodes de mesure de la digestibilité fécale (respectivement $21 \pm 3,20 \pm 1$ et $24 \pm 1 \%$ avec les aliments témoin, pois et soja). Le gain de poids vif, mesuré après la fin des transitions alimentaires, a été satisfaisant (respectivement $922 \pm 119,802 \pm 47$ et $785 \pm 77$ ), compte tenu des manipulations subies par les animaux et de la vacuité plus grande du tube digestif lors des 
pesées finales par rapport aux pesées initiales, puisque les digesta n'ont pas été réintroduits.

\section{Digestibilité apparente globale}

A la fin de l'iléon, la digestibilité apparente de la matière organique, de l'azote, de l'extractif non azoté, du calcium et du phosphore est moins élevée avec les aliments pois et soja qu'avec l'aliment témoin (Tableau II). Toutes les différences sont significatives pour l'aliment pois, tandis que seules celles concernant l'azote, le calcium et le phosphore le sont pour l'aliment soja. La digestibilité de l'extractif non azoté de l'aliment pois est significativement inférieure à celle de l'aliment soja; il en résulte également des

Tableau II. Digestibilités apparentes iléale et fécale (moyennes et écarts types des moyennes).

\begin{tabular}{|c|c|c|c|c|c|c|}
\hline \multirow{2}{*}{$\begin{array}{l}\text { Aliment } \\
\text { Niveau }\end{array}$} & \multicolumn{2}{|c|}{ Témoin } & \multicolumn{2}{|c|}{ Pois } & \multicolumn{2}{|c|}{ Soja } \\
\hline & lléal & Fécal & lléal & Fécal & lléal & Fécal \\
\hline Matière organique & $\begin{array}{l}0,923 \mathrm{a} \\
0,010\end{array}$ & $\begin{array}{l}0,978 A^{* *} \\
0,002\end{array}$ & $\begin{array}{l}0,849 \mathrm{~b} \\
0,026\end{array}$ & $\begin{array}{l}0,901 B^{* *} \\
0,012\end{array}$ & $\begin{array}{l}0,913 \mathrm{a} \\
0,005\end{array}$ & $\begin{array}{l}0,941 C^{*} \\
0,005\end{array}$ \\
\hline Azote & $\begin{array}{l}0,946 \text { a } \\
0,006\end{array}$ & $\begin{array}{l}0,973 A^{\star \star *} \\
0,003\end{array}$ & $\begin{array}{l}0,921 b \\
0,009\end{array}$ & $\begin{array}{l}0,921 \mathrm{~B} \\
0,009\end{array}$ & $\begin{array}{l}0,914 b \\
0,007\end{array}$ & $\begin{array}{l}0,936 B^{*} \\
0,005\end{array}$ \\
\hline Lipides & $\begin{array}{l}0,892 \\
0,020\end{array}$ & $\begin{array}{l}0,949 A^{*} \\
0,003\end{array}$ & $\begin{array}{l}0,885 \\
0,024\end{array}$ & $\begin{array}{l}0,893 \mathrm{~B} \\
0,019\end{array}$ & $\begin{array}{l}0,850 \\
0,019\end{array}$ & $\begin{array}{l}0,860 \mathrm{~B} \\
0,014\end{array}$ \\
\hline Cendres & $\begin{array}{l}0,691 \\
0,058\end{array}$ & $\begin{array}{l}0,933 A^{* *} \\
0,007\end{array}$ & $\begin{array}{l}0,727 \\
0,027\end{array}$ & $\begin{array}{l}0,822 B^{* *} \\
0,014\end{array}$ & $\begin{array}{l}0,748 \\
0,011\end{array}$ & $\begin{array}{l}0,824 B^{* *} \\
0,013\end{array}$ \\
\hline Extractif non azoté & $\begin{array}{l}0,925 \mathrm{~A} \\
0,018\end{array}$ & $\begin{array}{l}0,992 A^{*} \\
0,002\end{array}$ & $\begin{array}{l}0,797 \text { B } \\
0,043\end{array}$ & $\begin{array}{l}0,917 \mathrm{~B}^{*} \\
0,016\end{array}$ & $\begin{array}{l}0,938 \mathrm{~A} \\
0,005\end{array}$ & $\begin{array}{l}0,978 A^{* *} \\
0,005\end{array}$ \\
\hline Calcium & $\begin{array}{l}0,555 \text { a } \\
0,037\end{array}$ & $\begin{array}{l}0,817 A^{* *} \\
0,021\end{array}$ & $\begin{array}{l}0,355 \mathrm{~b} \\
0,062\end{array}$ & $\begin{array}{l}0,500 \mathrm{~B} \\
0,067\end{array}$ & $\begin{array}{l}0,372 \mathrm{~b} \\
0,036\end{array}$ & $\begin{array}{l}0,513 \mathrm{~B}^{*} \\
0,031\end{array}$ \\
\hline Phosphore & $\begin{array}{l}0,963 \mathrm{~A} \\
0,008\end{array}$ & $\begin{array}{l}0,980 \mathrm{~A} \\
0,003\end{array}$ & $\begin{array}{l}0,902 \mathrm{~B} \\
0,016\end{array}$ & $\begin{array}{l}0,890 \mathrm{~B} \\
0,008\end{array}$ & $\begin{array}{l}0,877 \text { B } \\
0,008\end{array}$ & $\begin{array}{l}0,863 \mathrm{~B} \\
0,010\end{array}$ \\
\hline Magnésium & $\begin{array}{l}0,537 \\
0,045\end{array}$ & $\begin{array}{l}0,706 a^{*} \\
0,033\end{array}$ & $\begin{array}{l}0,370 \\
0,055\end{array}$ & $\begin{array}{l}0,520 \mathrm{~b} \\
0,055\end{array}$ & $\begin{array}{l}0,477 \\
0,027\end{array}$ & $\begin{array}{l}0,517 \text { b } \\
0,035\end{array}$ \\
\hline Sodium & $\begin{array}{l}0,530 \\
0,074\end{array}$ & $\begin{array}{l}0,986 a^{* *} \\
0,003\end{array}$ & $\begin{array}{l}0,565 \\
0,046\end{array}$ & $\begin{array}{l}0,952 b^{* *} \\
0,009\end{array}$ & $\begin{array}{l}0,435 \\
0,007\end{array}$ & $\begin{array}{l}0,965 b^{* *} \\
0,005\end{array}$ \\
\hline Potassium & $\begin{array}{l}0,791 \\
0,065\end{array}$ & $\begin{array}{l}0,990 A^{\star *} \\
0,001\end{array}$ & $\begin{array}{l}0,886 \\
0,009\end{array}$ & $\begin{array}{l}0,946 B^{* *} \\
0,011\end{array}$ & $\begin{array}{l}0,888 \\
0,010\end{array}$ & $\begin{array}{l}0,979 A^{* *} \\
0,002\end{array}$ \\
\hline
\end{tabular}

$A, B, C, a, b, C: a ̀$ un niveau donné (iléal ou fécal), les moyennes accompagnées uniquement de lettres différentes sont significativement différentes (majuscules: $P<0,01$; minuscules : $P<0,05$ ). $"{ }^{\prime}, "$ : différences significatives entre les digestibilités iléale et fécale $(P<0,01$ et $P<0,05$, respectivement). 
valeurs moins élevées pour la digestibilité de la matière organique.

A la fin du tube digestif, la digestibilité apparente est très élevée pour tous les constituants de l'aliment témoin, magnésium et calcium exceptés (Tableau II). Les valeurs observées avec les aliments pois et soja sont significativement moins élevées qu'avec l'aliment témoin, sauf pour l'extractif non azoté et le potassium avec l'aliment soja. La matière organique, l'extractif non azoté et le potassium sont moins digestibles avec l'aliment pois qu'avec l'aliment soja.

Dans le cas de l'aliment témoin, la digestibilité fécale est toujours significativement plus élevée que la digestibilité iléale, sauf pour le phosphore. Les différences sont modérées pour l'azote, la matière organique, les lipides, l'extractif non azoté et la matière sèche; en revanche, elles sont beaucoup plus importantes pour les minéraux autres que le phosphore. Des différences allant dans le même sens sont également observées avec les deux autres aliments, sauf pour les lipides, ainsi que pour l'azote dans le cas de l'aliment pois.

\section{Acides aminés}

Les protéines des digesta et celles des fèces (Tableau III) contiennent davantage de thréonine, de sérine, de glycine, d'alanine et de cystine que les protéines totales des aliments (Tableau I). En revanche, elles sont généralement moins riches en méthionine, lysine et histidine. II en résulte des distances du $\chi^{2}$ élevées entre les compositions des protéines alimentaires étudiées d'une part, et celles des digesta (de 140 à 259) ou des fèces (de 168 à 299) d'autre part. Les digesta sont mieux pourvus que les fèces en thréonine, acide glutamique et proline, mais moins riches en alanine, méthionine, isoleucine, leucine, tyrosine et arginine. Par conséquent, les distances du $\chi^{2}$ entre les digesta et les fèces correspondantes sont importantes, au moins avec les aliments témoin et soja (respectivement 93 et 123 , mais seulement 50 avec l'aliment pois).

Avec l'aliment pois, les digesta iléaux sont significativement plus riches en acide aspartique qu'avec l'aliment témoin. Avec l'aliment soja, ils sont plus riches en isoleucine et leucine mais contiennent moins de sérine qu'avec l'aliment témoin. Les teneurs en sérine, cystine, lysine et histidine sont plus élevées, tandis que celles en isoleucine et leucine sont plus faibles avec l'aliment pois qu'avec l'aliment soja. Cependant, globalement, les différences restent minimes puisque les distances du $\chi^{2}$ entre les digesta sont comprises entre 17 et 24 . En revanche, les distances du $\chi^{2}$ sont relativement élevées entre les digesta et les produits endogènes (de 111 à 185) ou les bactéries (de 139 à 174). Dans le plan 1-2 de l'analyse factorielle des correspondances (AFC), les digesta sont regroupés dans une zone peu étendue se distinguant nettement de celles occupées respectivement par les produits endogènes, les bactéries et les aliments (Fig. 1).

La composition en acides aminés des fèces varie très peu suivant que les veaux reçoivent l'aliment témoin ou l'aliment pois (distance du $\chi^{2}=14$ ). De même, les écarts sont faibles avec les aliments pois et soja (distance du $\chi^{2}=35$ ), mais ils sont un peu plus nets avec les aliments témoin et soja (distance du $\chi^{2}=57$ ). La composition en acides aminés des fèces est voisine de celle des bactéries (distance du $\chi^{2}$ variant de 31 à 41 ). En revanche, elle est très différente de celle des protéines endogènes indigérées 
Tableau III. Composition en acides aminés (AA) des digesta, des fèces et des suppléments d'indigéré (\% des acides aminés dosés) (moyennes et écarts types des moyennes pour les digesta).

\begin{tabular}{|c|c|c|c|c|c|c|c|c|}
\hline \multirow{2}{*}{$\begin{array}{l}\text { Niveau } \\
\text { Aliment }\end{array}$} & \multicolumn{3}{|c|}{ lléon } & \multicolumn{3}{|c|}{ Fèces } & \multicolumn{2}{|c|}{$S I^{1}$} \\
\hline & Témoin & Pois & Soja & Témoin & Pois & Soja & Pois & Soja \\
\hline A. aspartique & $8,79 \pm 0,46 a$ & $10,46 \pm 0,39 b$ & $10,28 \pm 0,35 b$ & 11,74 & 10,77 & 9,40 & 13,18 & 11,04 \\
\hline Thréonine & $8,66 \pm 0,36$ & $8,39 \pm 0,50$ & $7,48 \pm 0,12$ & 6,47 & 6,69 & 5,70 & 8,26 & 6,67 \\
\hline Sérine & $5,83 \pm 0,19 a$ & $5,88 \pm 0,21 a$ & $5,18 \pm 0,15 b$ & 5,58 & 5,80 & 5,17 & 6,05 & 4,55 \\
\hline A. glutamique & $18,02 \pm 0,90$ & $17,23 \pm 1,85$ & $19,22 \pm 0,73$ & 13,93 & 13,46 & 12,31 & 13,78 & 20,65 \\
\hline Proline & $7,52 \pm 0,51 \mathrm{a}$ & $6,10 \pm 0,22 b$ & $6,27 \pm 0,21 b$ & 5,16 & 5,33 & 4,55 & 3,99 & 5,34 \\
\hline Glycine & $5,32 \pm 0,12$ & $5,34 \pm 0,14$ & $5,28 \pm 0,09$ & 4,90 & 5,23 & 5,78 & 5,35 & 5,31 \\
\hline Alanine & $5,80 \pm$ & $5,68 \pm 0,17$ & $5,87 \pm 0,24$ & 6,56 & 7,07 & 7,66 & 5,49 & 5,87 \\
\hline Valine & $6,20 \pm 0,09$ & $6,09 \pm 0,19$ & $6,10 \pm 0,09$ & 6,05 & 6,43 & 7,40 & 6,08 & 6,03 \\
\hline Cystine & $3,16 \pm 0,16 a$ & $3,48 \pm 0,16 \mathrm{~A}$ & $2,72 \pm 0,10 \mathrm{Bb}$ & b 2,98 & 3,23 & 2,67 & 4,30 & 2,39 \\
\hline Méthionine & $1,22 \pm 0,08$ & $1,41 \pm 0,06$ & $1,25 \pm 0,05$ & 1,87 & 2,00 & 2,35 & 1,68 & 1,27 \\
\hline Isoleucine & $3,82 \pm 0,14 \mathrm{~A}$ & $3,71 \pm 0,15 \mathrm{~A}$ & $4,54 \pm 0,10 B$ & 5,31 & 4,44 & 5,71 & 3,64 & 5,03 \\
\hline Leucine & $7,04 \pm 0,18 a$ & $6,78 \pm 0,33 \mathrm{~A}$ & $7,90 \pm 0,17 \mathrm{Bb}$ & b 7,32 & 8,41 & 10,58 & 6,36 & 8,51 \\
\hline Tyrosine & $2,86 \pm 0,20$ & $2,43 \pm 0,11$ & $2,61 \pm 0,10$ & 3,93 & 3,23 & 3 & 1,71 & 2,50 \\
\hline Phénylalanine & $4,63 \pm 0,49$ & $4,47 \pm 0,47$ & $4,74 \pm 0,17$ & 4,67 & 5,10 & 5,37 & 4,65 & 4,66 \\
\hline Lysine & $5,68 \pm 0,46 a$ & $6,65 \pm 0,21 b$ & $5,37 \pm 0,20 a$ & 6,66 & 6,20 & 5,90 & 8,54 & 5,25 \\
\hline Histidine & $2,14 \pm 0,08 a b$ & $2,22 \pm 0,02 a$ & $1,97 \pm 0,06 \mathrm{~b}$ & 1,94 & 2,11 & 1,89 & 2,38 & 1,85 \\
\hline Arginine & $3,33 \pm 0,22$ & $3,78 \pm 0,14$ & $3,23 \pm 0,12$ & 4,96 & 4,51 & 3,90 & 4,57 & 3,08 \\
\hline \multicolumn{8}{|l|}{ Somme des AA } & - \\
\hline \multicolumn{9}{|c|}{$N$ des AA dosés } \\
\hline
\end{tabular}

A, B, a, b : les moyennes accompagnées uniquement de lettres différentes sont significativement différentes (majuscules : $P<0,01$; minuscules : $P<0,05$ ); ${ }^{1}$ supplément d'indigéré iléal dû à l'aliment pois ou soja, par rapport à l'aliment témoin, pour une même quantité de matière sèche ingérée; ${ }^{2} \mathrm{~N}$ amidé de l'asparagine et de la glutamine exclu.

(distance du $\chi^{2}$ variant de 202 à 342 visà-vis des fèces d'agneau axénique).

A la fin de l'iléon, la digestibilité apparente de l'azote des acides aminés dosés est supérieure de 0,02 en moyenne à celle de l'azote total (Tableau IV). Pour les acides aminés indispensables et semiindispensables, la digestibilité est systématiquement inférieure à la moyenne pour la cystine et la thréonine. En revanche, c'est l'inverse pour la méthionine et la tyrosine. La digestibilité des acides aminés est systématiquement moins élevée avec les aliments pois et soja qu'avec l'aliment témoin. Les différences sont significatives, sauf pour la cystine et l'arginine avec les aliments pois et soja, plus la leucine, la tyrosine et la phénylalanine avec l'aliment pois. Les valeurs obtenues sont significativement plus élevées avec l'aliment pois qu'avec l'aliment soja pour la méthionine, l'isoleucine, la leucine, la tyrosine et l'histidine. 


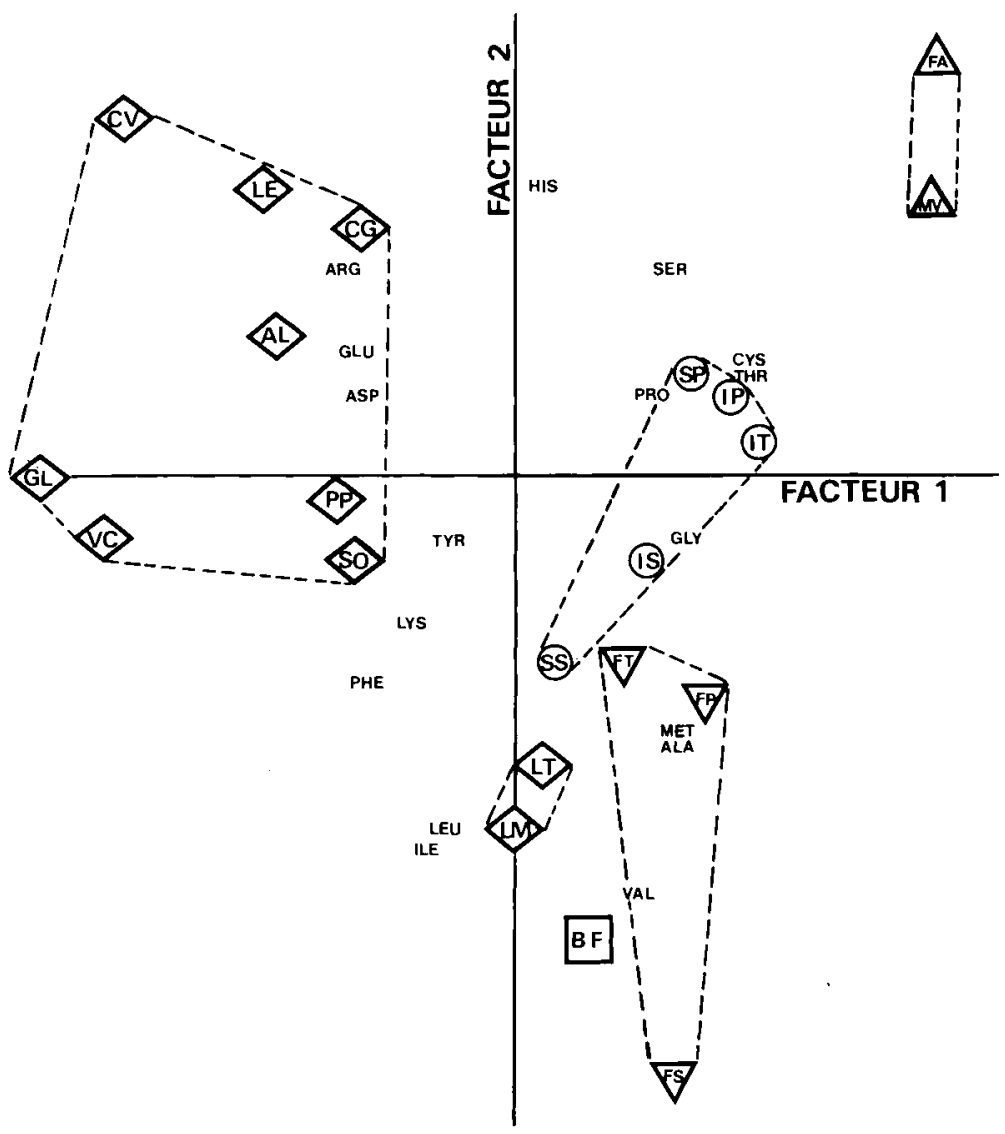

Fig. 1. Comparaison de la composition en acides aminés (\% de la somme des acides aminés dosés) des protéines des digesta iléaux $(O)$ et des fèces $(\nabla)$ avec celle des protéines alimentaires $(0)$, endogènes $(\Delta)$ et bactériennes (D). Chaque point est la projection d'une protéine sur le plan principal (1-2) de l'analyse factorielle des correspondances. Les 17 acides aminés sont également projetés. Les axes 1 et 2 contribuent respectivement pour 36 et $18 \%$ à l'inertie totale. I, F : digesta iléaux (I) et fèces $(F)$ avec les aliments Témoin (T), Pois (P) ou Soja (S); SP, SS : supplément d'indigéré iléal dû à l'aliment Pois ou Soja, par rapport à l'aliment Témoin; LT, LM : lait et lactosérum; $\mathrm{PP}, \mathrm{AL}, \mathrm{LE}, \mathrm{VC}, \mathrm{CV}$ : pois prégélatinisé, albumines, légumine, viciline et conviciline; SO, GL, CG : isolat de soja, glycinine et B-conglycinine; FA, MV : fèces d'agneau axénique et méconium de veau; BF : bactéries fécales de porc et de mouton (moyenne). Pour les références, cf. Matériel et Méthodes.

Au niveau fécal, les principales différences observées à la fin de l'iléon dans la digestibilité apparente entre les acides aminés individuels et l'azote des acides aminés dosés subsistent, sauf pour la tyrosine. De même, la digestibilité de tous les acides aminés reste systématiquement plus élevée avec l'aliment témoin. En revanche, les différences observées entre les aliments pois et soja 
Tableau IV. Digestibilités apparentes iléale et fécale des acides aminés (AA) (moyennes et écarts types des moyennes au niveau iléal).

\begin{tabular}{|c|c|c|c|c|c|c|}
\hline \multirow{2}{*}{$\begin{array}{l}\text { Aliment } \\
\text { Niveau }\end{array}$} & \multicolumn{2}{|c|}{ Témoin } & \multicolumn{2}{|l|}{ Pois } & \multicolumn{2}{|l|}{ Soja } \\
\hline & lléal & Fécal & Iléal & Fécal & lléal & Fécal \\
\hline A. aspartique & $0,963 \pm 0,005 \mathrm{Aa}$ & 0,964 & $0,943 \pm 0,008 b$ & 0,932 & $0,928 \pm 0,004 \mathrm{~B}$ & 0,954 \\
\hline Thréonine & $0,942 \pm 0,006 \mathrm{Aa}$ & 0,968 & $0,900 \pm 0,017 b$ & 0,909 & $0,871 \pm 0,007 \mathrm{~B}$ & 0,932 \\
\hline Sérine & $0,959 \pm 0,006 \mathrm{Aa}$ & 0,972 & $0,934 \pm 0,011 \mathrm{~b}$ & 0,926 & $0,913 \pm 0,005 \mathrm{~B}$ & 0,940 \\
\hline A. glutamique & $0,973 \pm 0,003 \mathrm{Aa}$ & 0,984 & $0,956 \pm 0,005 \mathrm{Ab}$ & 0,958 & $0,924 \pm 0,006 \mathrm{~B}$ & 0,966 \\
\hline Proline & $0,975 \pm 0,003 \mathrm{Aa}$ & 0,988 & $0,957 \pm 0,008 \mathrm{Ab}$ & 0,957 & $0,911 \pm 0,006 \mathrm{~B}$ & 0,955 \\
\hline Glycine & $0,907 \pm 0,011$ & 0,937 & $0,902 \pm 0,014 a b$ & 0,889 & $0,892 \pm 0,006$ & 0.918 \\
\hline Alanine & $0,939 \pm 0,007 \mathrm{a}$ & 0,950 & $0,920 \pm 0,012 a b$ & 0,885 & $0,898 \pm 0,009 b$ & 0,908 \\
\hline Valine & $0,972 \pm 0,003 \mathrm{Aa}$ & 0,980 & $0,948 \pm 0,008 \mathrm{Ab}$ & 0,937 & $0,919 \pm 0,005 \mathrm{~B}$ & 0,931 \\
\hline Cystine & $0,891 \pm 0,015$ & 0,926 & $0,828 \pm 0,031$ & 0,821 & $0,865 \pm 0,011$ & 0,908 \\
\hline Méthionine & $0,987 \pm 0,002 \mathrm{Aa}$ & 0,986 & $0,976 \pm 0,004 b$ & 0,960 & $0,966 \pm 0,003 \mathrm{Bc}$ & 0,956 \\
\hline Isoleucine & $0,978 \pm 0,002 \mathrm{Aa}$ & 0,978 & $0,962 \pm 0,006 b$ & 0,948 & $0,936 \pm 0,004 \mathrm{BC}$ & 0,944 \\
\hline Leucine & $0,975 \pm 0,004 \mathrm{~A}$ & 0,981 & $0,961 \pm 0,007 \mathrm{~A}$ & 0,944 & $0,928 \pm 0,004 \mathrm{~B}$ & 0,933 \\
\hline Tyrosine & $0,978 \pm 0,003 \mathrm{~A}$ & 0,978 & $0,968 \pm 0,006 \mathrm{~A}$ & 0,952 & $0,937 \pm 0,005 B$ & 0,938 \\
\hline Phénylalanine & $0,965 \pm 0,008 \mathrm{a}$ & 0,975 & $0,946 \pm 0,013 a b$ & 0,933 & $0,922 \pm 0,004 b$ & 0,938 \\
\hline Lysine & $0,976 \pm 0,004 \mathrm{Aa}$ & 0,979 & $0,955 \pm 0,007 \mathrm{~b}$ & 0,952 & $0,942 \pm 0,005 \mathrm{~B}$ & 0,956 \\
\hline Histidine & $0,972 \pm 0,004 \mathrm{Aa}$ & 0,982 & $0,954 \pm 0,007 b$ & 0,950 & $0,934 \pm 0,004 \mathrm{Bc}$ & 0,956 \\
\hline Arginine & $0,967 \pm 0,005$ & 0,965 & $0,961 \pm 0,006$ & 0,946 & $0,958 \pm 0,003$ & 0,965 \\
\hline $\begin{array}{l}\mathrm{N} \text { des } \mathrm{AA} \\
\text { dosés } 1\end{array}$ & $0,967 \pm 0,004 \mathrm{Aa}$ & 0,975 & $0,948 \pm 0,008 b$ & 0,939 & $0,926 \pm 0,004 \mathrm{Bc}$ & 0,947 \\
\hline
\end{tabular}

$A, B, C, a, b, c$ : les moyennes accompagnées uniquement de lettres différentes sont significativement differentes (majuscules : $P<0,01$; minuscules : $P<0,05$ ); ${ }^{1} \mathrm{~N}$ amidé de l'asparagine et de la glutamine exclu.

s'estompent fortement, sauf pour la cystine dont la digestibilité devient inférieure de 0,09 avec l'aliment pois.

Exprimées par $\mathrm{kg}$ de matière sèche ingérée, les quantités d'acides aminés indigérés sont toujours plus élevées avec les aliments pois et soja qu'avec l'aliment témoin, indépendamment des variations des quantités ingérées. La quantité totale d'azote sous forme d'acides aminés est respectivement multipliée par 1,6 et 2,4 à la fin de l'iléon et par 2,6 et 2,3 à la fin du tube digestif. II n'apparaît d'indisponibilité importante pour aucun acide aminé particulier, puisque le coefficient multiplicateur le plus élevé à la fin de l'iléon est de 1,9 pour la lysine de l'aliment pois et de 2,9 pour l'isoleucine de l'aliment soja.

\section{Légumine}

La légumine a été en grande partie dénaturée au cours de la prégélatinisation puisque la partie immunoréactive ne correspond plus qu'à $1,1 \%$ des matières azotées de la farine, au lieu d'environ 20 à $25 \%$ dans la plupart des farines crues (Guéguen \& Barbot, 1988). La légumine est détectée sous forme immunoréactive dans les digesta iléaux et les fèces, mais 
elle n'y représente respectivement que $1,1 \pm 0,7$ et $0,2 \pm 0,1 \%$ de la quantité ingérée.

\section{DISCUSSION}

\section{Digestibilité apparente}

L'augmentation de la digestibilité apparente de l'azote observée avec l'aliment témoin après passage dans le gros intestin est en accord avec les données publiées avec des aliments similaires (Van Weerden et al., 1977; Guilloteau \& Toullec, 1980; Guilloteau et al, 1986). L'augmentation concernant l'aliment soja est voisine de celle obtenue par Guilloteau et al. (1986) avec un concentrat de soja très digestible. Avec l'aliment pois, une part plus importante des glucides échappe à la digestion dans l'intestin grêle; il en résulte un accroissement des fermentations et de la quantité d'azote incorporé dans les corps bactériens (Ørskov et al., 1970), ce qui empêche l'augmentation de la digestibilité apparente de l'azote dans le gros intestin.

En admettant que la digestibilité de l'azote du lait soit la même dans les aliments témoin et pois, la digestibilité calculée pour l'azote du pois serait de 0,87 à la fin de l'iléon, mais seulement de 0,82 au niveau fécal. Seules les matières azotées absorbées dans l'intestin grêle sont intéressantes pour l'animal; en effet, celles qui sont absorbées dans le gros intestin sont essentiellement sous forme d'ammoniac (Zebrowska et al., 1978). Les différences observées au niveau iléal permettent donc de classer les sources de protéines avec davantage de précision que celles obtenues au niveau fécal. La digestibilité apparente de 0,87 trouvée à la fin de l'iléon pour l'azote du pois est inférieure à celle de l'azote du lait. Elle est cependant satisfaisante, si on la compare à celle observée au niveau fécal pour l'azote du tourteau de soja $(0,74$ d'après Paruelle et al., 1972) ou de la féverole crue $(0,72$ d'après Toullec et al., 1980). La digestibilité fécale de l'aliment pois est plus élevée que celle obtenue par Bell et al. (1974), chez des veaux plus jeunes ( 3 semaines), pour des aliments où la moitié des protéines était apportée par un concentrat protéique de pois ou une farine de pois dont l'amidon avait été hydrolysé (respectivement 0,79 et 0,73 au lieu de 0,88 pour l'aliment témoin).

A notre connaissance, la digestibilité apparente de l'azote du lactosérum n'a pas été mesurée à la fin de l'iléon. Nous l'avons donc estimée en multipliant la valeur obtenue au niveau fécal $(0,94)$ par Grongnet et al. (1981) par le rapport digestibilité iléale/digestibilité fécale trouvé ici, pour l'azote total du lait $(0,97)$. Dans ces conditions, la valeur calculée pour la digestibilité apparente de l'azote de l'isolat de soja serait de 0,91 à la fin de l'iléon et de 0,93 au niveau fécal. Ces valeurs sont plus élevées que celle observée à la fin du tube digestif pour un concentrat de soja préparé par extraction hydroalcoolique des oligosides $(0,87$ d'après Guilloteau et al., 1986), ce qui pourrait être dû à l'absence de glucides indigestibles dans l'isolat. L'isolat est aussi digestible que les concentrats protéiques de lactosérum $(0,89$ à 0,94 d'après Grongnet et al., 1981), les bactéries cultivées sur méthanol $(0,91$ d'après Guilloteau \& Toullec, 1980) et les hydrolysats de poisson $(0,91$ d'après Paruelle et al., 1974).

En accord avec les données bibliographiques obtenues avec des 
aliments contenant des protéines de lait, de soja, de poisson ou de bactéries (Van Weerden et al., 1977; Guilloteau \& Toullec, 1980; Guilloteau et al., 1986), la digestion des lipides est pratiquement achevée à la fin de l'iléon dans le cas des aliments pois et soja. \| est donc surprenant de voir augmenter la digestibilité des lipides au cours de leur passage dans le gros intestin, avec l'aliment témoin. Une part notable de l'extractif non azoté (ENA) disparaît dans le gros intestin, surtout avec l'aliment pois. Les acides gras volatils et l'acide lactique formés sont bien utilisés par le veau (Vermorel \& Patureau-Mirand, 1978). Avec les aliments témoin et soja, l'ENA présent dans les digesta iléaux est essentiellement d'origine endogène puisque le lactose et les petites quantités d'amidons traités apportés par les aliments sont totalement digérés dans l'intestin grêle (Besle et al., 1981). A ce niveau, la digestibilité plus faible de l'aliment pois est due à sa teneur plus élevée en amidon (environ $17 \%$ de la matière sèche, au lieu de 6 dans les deux autres aliments), mais aussi à la présence de cellulose et d'oligosides (représentant respectivement environ 1 et $3 \%$ de la matière sèche). Lorsqu'il est présent en proportion importante dans le régime, une part notable de l'amidon peut échapper à la digestion dans l'intestin grêle et être dégradée dans le gros intestin (Assan \& Thivend, 1976). La cellulose et les oligosides ne sont pas attaqués par les enzymes du veau. Une partie des oligosides disparaît dans l'intestin grêle sous l'action des bactéries, ainsi que par absorption, mais celle qui subsiste dans les digesta est fermentée en totalité dans le gros intestin (Besle et al., 1981). En admettant que la cellulose soit totalement indigérée, la digestibilité apparente de l'amidon de pois serait au maximum de 0,84 dans l'ensemble du tube digestif.
Dans l'ensemble du tube digestif, les résultats concernant la digestibilité apparente des minéraux de l'aliment témoin sont en accord avec ceux obtenus par Guéguen \& Mathieu (1962) dans le cas du lait entier. Avec les aliments pois et soja, la résorption moins forte du calcium et du magnésium s'explique au moins en partie par des différences dans les formes chimiques et par la croissance moins rapide des animaux. Le phosphore est également plus abondant et se trouve en partie sous forme phytique; cela pourrait contribuer à réduire son absorption et celle du calcium et du magnésium. Conformément aux données bibliographiques (Thivend \& Toullec, 1977), l'absorption du phosphore est apparemment achevée à la fin de l'iléon, tandis que des proportions notables de potassium et surtout de magnésium et de sodium sont absorbées dans le gros intestin (en moyenne 12, 20 et $48 \%$ des quantités totales absorbées). En revanche, l'importance de l'absorption du calcium dans cet organe est inattendue ( $30 \%$ de la quantité totale absorbée).

\section{Origine des protéines indigérées}

Avec l'aliment témoin, la composition en acides aminés des digesta iléaux est voisine de celles rapportées par Guilloteau et al. (1980 et 1986) avec du lait. L'AFC suggère que la composition en acides aminés des digesta est comprise entre celle des produits endogènes et celle des bactéries (Fig. 1). Les calculs effectués par itération montrent qu'un mélange théorique de protéines provenant pour $58 \%$ des fèces d'agneau axénique et $42 \%$ des bactéries fécales ressemble davantage aux protéines des digesta que chacune des deux sources prises séparément (distance du $\chi^{2}$ de 52 
au lieu de 111 et 174). Bien qu'un mélange théorique comprenant 51,28 et $21 \%$ de protéines de fèces d'agneau axénique, de bactéries et d'aliment témoin soit plus proche des digesta (distance du $\chi^{2}=40$ ), il est peu probable qu'une quantité notable de protéines de lait échappe à la digestion dans l'intestin grêle. En effet, nos résultats antérieurs (Guilloteau et al., 1986) ont montré que la composition des digesta iléaux variait peu dans la journée lorsque les protéines alimentaires provenaient du lait, mais beaucoup lorsqu'elles étaient apportées par du soja ou du poisson. Avec ces deux dernières sources de protéines, la composition trouvée quand le débit était minimal était très proche de celle observée en moyenne avec le lait. Les protéines des digesta iléaux des veaux nourris au lait peuvent donc être considérées comme représentant le mélange de protéines endogènes et microbiennes échappant à la digestion dans l'intestin grêle.

Les faibles variations entraînées dans la composition en acides aminés des digesta iléaux en remplaçant l'aliment témoin par les aliments pois ou soja (distance du $\chi^{2} \leq 19$ par rapport aux digesta obtenus avec l'aliment témoin) suggèrent que peu de protéines alimentaires échappent à la digestion dans l'intestin grêle. Ainsi, la quantité de légumine immunoréactive présente dans les digesta obtenus avec l'aliment pois ne représente qu'une faible part de la quantité ingérée. De même, le mélange théorique de protéines de pois et de digesta obtenus avec l'aliment témoin qui ressemble le plus à celles des digesta correspondant à l'aliment pois (distance du $\chi^{2}=12$ ) ne contient que $13 \%$ de protéines de pois. Enfin, le supplément d'indigéré dû à l'aliment pois par rapport à l'aliment témoin (Tableau III) est plus proche des digesta obtenus avec ce dernier aliment que des diverses protéines du pois (Fig. 1). Cependant, aucun modèle simulant de manière satisfaisante la composition du supplément d'indigéré ne peut être constitué à partir des protéines alimentaires, endogènes et/ou bactériennes (distance du $\chi^{2} \geq 81$ ). Cela pourrait signifier que les fractions alimentaires indigérées ont des compositions en acides aminés différentes de celles des protéines entières du pois. Une autre hypothèse serait que le pois provoque une augmentation de certaines sécrétions endogènes de composition particulière. En se basant sur le mélange théorique de protéines de pois et de digesta correspondant à l'aliment témoin qui simule le mieux les protéines des digesta obtenus avec l'aliment pois, on peut estimer que ce dernier entraîne un accroissement d'environ $37 \%$ dans les quantités de protéines endogènes et bactériennes échappant à la digestion dans l'intestin grêle. Une part indéterminée des protéines bactériennes provient de la transformation des protéines du pois. Même en admettant que ce soit le cas pour la totalité des protéines bactériennes du supplément d'indigéré, la digestibilité réelle des protéines du pois serait très élevée (de l'ordre de 0,96 ) à la fin de l'iléon.

Avec l'aliment soja, les digesta iléaux et surtout le supplément d'indigéré (Tableau III) sont situés entre les digesta obtenus avec l'aliment témoin, l'isolat de soja et les bactéries, dans le plan 1-2 de l'AFC (Fig. 1). Cependant, la part des protéines bactériennes augmente probablement moins que celle des protéines alimentaires. En effet, dans le plan 1-3 de I'AFC (non montré), les digesta obtenus avec les aliments témoin et soja, le supplément d'indigéré et l'isolat de soja sont alignés dans cet ordre le long de l'axe 1, tandis que les bactéries s'en écartent le long de l'axe 3 (ces 2 axes 
rendent compte respectivement de 36 et $13 \%$ de l'inertie totale). De même, le mélange théorique de protéines de digesta obtenus avec l'aliment témoin, d'isolat de soja et de bactéries qui se rapproche le plus du supplément d'indigéré (distance du $\chi^{2}$ de 38 ) renferme 65 et $27 \%$ des deux premières protéines, mais seulement $8 \%$ de la troisième. Néanmoins, le supplément d'indigéré n'est pas plus riche en arginine que les protéines des digesta correspondant à l'aliment témoin. Comme nous l'avons déjà observé dans le cas d'un concentrat de soja traité à l'alcool (Guilloteau et al., 1986) et avec le pois, ce serait des fractions partiellement dégradées de protéines d'isolat qui échappent à la digestion dans l'intestin grêle. Au contraire, avec du tourteau de soja peu digestible, les protéines alimentaires indigérées ont une composition en acides aminés très voisine de celle des protéines totales du soja (Duvaux et al., 1989). L'aliment soja entraînerait donc un accroissement d'environ $90 \%$ dans les quantités de protéines endogènes et bactériennes parvenant à la fin de l'iléon. Le même raisonnement que celui appliqué dans le cas du pois conduit également à une valeur très élevée $(0,97)$ pour la digestibilité réelle des protéines de l'isolat de soja.

Conformément à nos observations antérieures (Patureau-Mirand et al., 1977; Guilloteau et al., 1986), la composition en acides aminés des fèces varie peu avec la nature des protéines alimentaires. Cela est dû à la prédominance des protéines bactériennes mais s'explique également, dans notre essai, par la faible variabilité existant déjà à la fin de l'iléon.

En conclusion, le remplacement des protéines du lait par celles d'une farine de pois prégélatinisée ou d'un isolat de soja entraîne une réduction modérée de la digestibilité apparente pour la matière organique, l'azote, les lipides et le phosphore, mais plus importante pour le calcium et le magnésium. L'augmentation de la quantité d'acides aminés échappant à la digestion dans l'intestin grêle semble être davantage due à un accroissement des pertes de protéines endogènes et bactériennes qu'à une digestibilité réelle incomplète. Les glucides du pois et les facteurs antinutritionnels résiduels présents dans le pois et le soja étudiés, ainsi que l'accélération de l'évacuation gastrique résultant de leur introduction dans l'aliment, n'entraînent que des effets dépressifs modérés. En particulier, il ne semble pas se produire de phénomènes allergiques caractérisés avec le pois, malgré la formation d'anticorps contre ses protéines (Nunes do Prado et al., 1989). Sous réserve d'essais complémentaires permettant de préciser leurs effets à plus long terme sur la croissance et la qualité de la carcasse, ces produits pourraient donc apporter une part notable des protéines dans les régimes de finition du veau de boucherie.

\section{REMERCIEMENTS}

A Jeannine Quillet pour avoir réuni les données bibliographiques, à la Coopérative de traitement des produits de la pêche et à Duquesne-Purina qui ont gracieusement fourni la farine de pois et l'isolat de soja.

\section{RÉFÉRENCES}

Assan B.E. \& Thivend P. (1976) Intestinal digestion of tropical starches by the preruminant calf. Proc. Nutr. Soc. 35, 104A-105A

Bell J.M., Royan G.F. \& Youngs C.G. (1974) Digestibility of pea protein concentrate and 
enzyme-treated pea flour in milk replacers for calves. Can. J. Anim. Sci. 54, 355-362

Besle J.M., Lassalas B. \& Thivend P. (1981) Digestion des glucides cytoplasmiques de la féverole par le veau préruminant. Reprod. Nutr. Dév. 21, 629-649

Combe E. (1976) Influence de la microflore intestinale sur la composition en acides aminés des fèces des agneaux. C. $R$. Séances Soc. Biol. Fil. 170, 787-793

Groy R.R.D., Gatehouse J.A., Tyler M. \& Boulter D. (1980) The purification and characterization of a third storage protein (convicilin) from the seeds of pea (Pisum sativum L.). Biochem. J. 191, 509-516

Duvaux C., Sissons J.W., Pedersen H.E., Guilloteau P. \& Toullec R. (1989) Digestion of allergenic soya protein in the preruminant calf. Reprod. Nutr. Dev. 29, suppl. (à paraître)

Grongnet J.F., Patureau-Mirand P., Toullec R. \& Prugnaud J. (1981) Utilisation des protéines du lait et du lactosérum par le jeune veau préruminant. Influence de l'âge et de la dénaturation des protéines du lactosérum. Ann. Zootech. 30, 443-464

Guéguen J. \& Barbot J. (1988) Quantitative and qualitative variability of pea (Pisum Sativum L.) protein composition. J. Sci. Food Agric. 42, 209-224

Guéguen J., Vu A.T. \& Schaeffer F. (1984) Large-scale purification and characterization of pea globulins. J. Sci. Food Agric. 35, 10241033

Guéguen L. \& Mathieu C.-M. (1962) L'utilisation des éléments minéraux de la ration par le veau. I. Influence du régime alimentaire. Ann. Zootech. 14, 231-245

Guilloteau P., Patureau-Mirand P., Toullec R. \& Prugnaud J. (1980) Digestion of milk protein and methanol grown bacteria protein in the preruminant calf. II. Amino acid composition of ileal digesta and faeces and blood levels of free amino-acids. Reprod. Nutr. Dév. 20, 615629

Guilloteau P., Sauvant D. \& Patureau-Mirand P. (1983) Methods of comparing amino acid composition of proteins : applications to undigested proteins in the preruminant calf. Ann. Nutr. Metab. 27, 457-469

Guilloteau P. \& Toullec R. (1980) Digestion of milk protein and methanol grown bacteria protein in the preruminant calf. I. Kinetics and balance in the terminal small intestine and faecal balance. Reprod. Nutr. Dév. 20, 601-613 Guilloteau P., Toullec R., Grongnet J.F., Patureau-Mirand P., Prugnaud J. \& Sauvant D. (1986) Digestion of milk, fish and soya-bean protein in the preruminant calf : flow of digesta, apparent digestibility at the end of the ileum and amino acid composition of ileal digesta. Br. J. Nutr. 55, 571-592

Gwiazda S., Schwenke K.D. \& Rutkowski A. (1980) Isolation and partial purification of proteins from pea (Pisum sativum L.). Nahrung 24, 939-950

Mason V.C. (1979) The quantitative importance of bacterial residues in the non-dietary faecal nitrogen in sheep. 1 . Methodology studies. $Z$. Tierphysiol. Tierernähr. Futtermittelkd. 41, 131139

Mason V.C., Just A. \& Bech-Andersen S. (1976) Bacterial activity in the hindgut of pigs. 2. Its influence on the apparent digestibility of nitrogen and amino-acids. $Z$. Tierphysiol. Tierernähr. Futtermittelkd. 36, 310-324

Nunes do Prado I., Toullec R., Lallès J.P., Guéguen J. \& Guilloteau P. (1989) Digestion des protéines de pois et de soja chez le veau préruminant. I. Taux circulants de nutriments, formation d'anticorps et perméabilité intestinale aux macromolécules. Reprod. Nutr. Dev. 29 (à paraître)

Ørskov E.R., Fraser C., Mason V.C. \& Mann S.O. (1970) Influence of starch digestion in the large intestine of sheep on caecal fermentation, caecal microflora and faecal nitrogen excretion. Br. J. Nutr. 24, 671-682

Paruelle J.L., Toullec R., Frantzen J.F. \& Mathieu C.M. (1972) Utilisation des protéines par le veau préruminant à l'engrais. I. Utilisation digestive des protéines de soja et des levures d'alcanes incorporées dans les aliments d'allaitement. Ann. Zootech. 21, 318-331

Paruelle J.L., Toullec R., Patureau-Mirand P. \& Mathieu C.M. (1974) Utilisation des protéines par le veau préruminant à l'engrais. Ann. Zootech. 23, 519-536

Patureau-Mirand P., Toullec R., Guilloteau P. \& Pion R. (1977) Influence de la nature des protéines alimentaires sur la composition en acides aminés des fèces du veau préruminant. Ann. Biol. Anim. Biochim. Biophys. 17, 71-83 
Thivend P. \& Toullec R. (1977) Les aliments d'allaitement. In : Le veau. (Mornet P. \& Espinasse J., eds) Maloine, Paris

Toullec R., Coroller J.Y., Patureau-Mirand P., Melcion J.P., Prugnaud J., Valdebouze P. \& Delort-Laval J. (1980) Influence des traitements technologiques sur l'utilisation des proténes de la féverole par le veau préruminant. Ann. Zootech. 29, 339-361

Toullec R., Guilloteau P., Patureau-Mirand P. \& Sissons J.W. (1983) Digestion and absorption of protein in the preruminant. In : Protein Metab. Nutr. I. (Arnal M., Pion R., Bonin D., eds) INRA Paris

Van Weerden E.J., Huisman J. \& Van Hellemond K.K. (1977) Veterrings fysiologisch onderzoek. Enkele unikkomsten ten aazien van het verterings proces in het maagdarmkanaal van het mestkalf. Landbouwkd. Tijdschr. 89, 217-224
Vermorel M. \& Patureau-Mirand P. (1978) Utilisation des produits terminaux de la digestion des glucides et des lipides par le veau préruminant. In : Le Veau de Boucherie. INRA publications, Versailles

Walker-Smith J.A. (1984) Cow's milk protein intolerance in infancy. In : Food Intolerance. (Chandra R.K., ed.) Elsevier, New York

Wolf W.J. (1972) Purification and properties of the proteins. In : Soybeans:Chemistry and Technology. (Smith A.K. \& Circle S.J., eds) AVI Publishing company, Westport

Zebrowska T., Buraczewska L. \& Horaczynski $H$. (1978) Apparent digestibility of nitrogen and amino-acids and utilization of protein given orally or introduced into the large intestine of pigs. Rocz. Nauk Roln. Ser. B. Zootech. 99, 99105 